\title{
DECOMPOSABILITY OF FINITELY PRESENTED MODULES
}

\author{
R. B. WARFIELD, JR.
}

\begin{abstract}
It is proved that a commutative ring with 1 has the property that every finitely presented module is a summand of a direct sum of cyclic modules if and only if it is locally a generalized valuation ring. A Noetherian ring has this property if and only if it is a direct product of a finite number of Dedekind domains and an Artinian principal ideal ring. Any commutative local ring which is not a generalized valuation ring has finitely presented indecomposable modules requiring arbitrarily large numbers of generators.
\end{abstract}

All rings in this paper are commutative, associative and have an identity. A commutative ring $R$ is a generalized valuation ring if it satisfies one of the following three equivalent conditions:

(i) for any two elements $a$ and $b$, either $a$ divides $b$ or $b$ divides $a$;

(ii) the ideals of $R$ are linearly ordered by inclusion;

(iii) $R$ is a local ring (i.e. it has only one maximal ideal) and every finitely generated ideal is principal.

A valuation ring in the usual sense is just a generalized valuation ring which is a domain. The rings which are locally generalized valuation rings might be thought of as extremely generalized Dedekind rings. The work reported here is a continuation of $[6, \S 2]$.

1. Modules over generalized valuation rings. A submodule $A$ of an $R$-module $B$ is relatively divisible if for all $r \in R, r A=A \cap r B$. A module $P$ is RD-projective if for every short exact sequence $0 \rightarrow A \rightarrow B$ $\rightarrow C \rightarrow 0$ with $A$ relatively divisible in $B$, the natural map $\operatorname{Hom}(P, B)$ $\rightarrow \operatorname{Hom}(P, C)$ is surjective. By [6, Proposition 2], an $R$-module (over an arbitrary ring) is RD-projective if and only if it is a summand of a direct sum of cyclic modules of the form $R / R r, r \in R$. (Such cyclic modules are called cyclically presented or CP modules.) Relatively divisible submodules are sometimes called pure submodules, and the $\mathrm{RD}$-projectives "pure-projective," but the terminology is not uniform.

A module $M$ is said to be finitely presented if for some finitely generated free module $F$ and finitely generated submodule $K$ of $F$, $M=F / K$. An important result about these modules is that if $M$ is

Received by the editors August 4, 1969.

A MS Subject Classifications. Primary 1340, 1350; Secondary 1325, 1395.

Key Words and Phrases. Indecomposable modules, generalized valuation rings, direct sums of cyclic modules. 
finitely presented and $M=F / G$, where $F$ is a finitely generated free module, then $G$ is also finitely generated (Bourbaki, Algèbre commutative, Chapter I, p. 37). The special case of this which we will use below is that a finitely presented cyclic module over a generalized valuation ring is cyclically presented.

THEOREM 1. If $M$ is a finitely presented module over a generalized valuation ring then $M$ is a direct sum of $\mathrm{CP}$ modules.

REMARK. This proof corrects what we believe to be a flaw in the proofs of [6, Theorem 1] and [3, Theorem 14], since in both of these papers it is taken as obvious that if an element is chosen with minimal order ideal then it generates a relatively divisible submodule.

Proof. Let $m$ be the maximal ideal of $R$ and $y_{1}, \cdots, y_{n}$ a basis for the $R / \mathrm{m}$-vector space $M / \mathrm{m} M$. If $x_{1}, \cdots, x_{n}$ are elements of $M$ with $x_{i}+\mathfrak{m} M=y_{i}$, then the $x_{i}$ generate $M$. We remark first that one of the basis elements $y_{1}, \cdots, y_{n}$ (say $y_{1}$ ) has the property that any element $x$ in $M$ with $x+m M=y_{1}$ has order ideal equal to the annihilator ideal of $M$. (If this were not the case, we could choose a set of generators $x_{1}, \cdots, x_{n}$ as above such that the order ideals of the $x_{i}$ were all greater than the annihilator ideal of $M$. Since the ideals of $R$ are linearly ordered, and the annihilator ideal of $M$ is the intersection of the order ideals of the $x_{i}$, this is impossible.) If $y_{1}$ is chosen in this way and $x_{1}$ is chosen so that $x_{1}+\mathfrak{m} M=y_{1}$, then the submodule $\left(x_{1}\right)$ generated by $x_{1}$ is relatively divisible. (To see this, suppose $r x_{1}=s z$, where $z \in M$ and $s$ does not divide $r$. Then $s=r t$ for some $t \in \mathfrak{m}$. If, then, $x_{1}^{*}=x_{1}-t z$, then $x_{1}^{*}+\mathrm{m} M=y_{1}$ and $r x_{1}^{*}=0$, so (by our condition on $\left.y_{1}\right) r x_{1}=0$. Hence $r x_{1}$ is divisible by $s$, which shows that $\left(x_{1}\right)$ is a relatively divisible submodule.)

We now look at the sequence $0 \rightarrow\left(x_{1}\right) \rightarrow M \rightarrow M /\left(x_{1}\right) \rightarrow 0$. By induction, $M /\left(x_{1}\right)$ is a direct sum of CP modules, and is therefore RD-projective. Hence $M \cong\left(x_{1}\right) \oplus M /\left(x_{1}\right)$, from which it follows that $\left(x_{1}\right)$ is also finitely presented and therefore a CP module, so Theorem 1 is proved.

THEOREM 2. Let $R$ be a local ring which is not a generalized valuation ring. Then for any $n>0$, there are finitely presented modules which are indecomposable and which cannot be generated by fewer than $n$ elements.

REMARK. The technique used here was motivated by D. Higman's construction of indecomposable group representations [2].

Proof. Let $a$ and $b$ be elements of $R$, neither dividing the other. By taking a suitable quotient ring, we may assume $(a) \cap(b)=0$, and $\mathfrak{m}(a)=\mathfrak{m}(b)=0$ (where $\mathfrak{m}$ is the maximal ideal). Let $F$ be a free 
module with generators $x_{1}, \cdots, x_{n}$, let $K$ be the submodule generated by $a x_{1}-b x_{2}, \cdots, a x_{n-1}-b x_{n}$, and let $M=F / K$. Clearly $M / \mathfrak{m} M$ $\cong F / \mathfrak{m} F$, so $M$ cannot be generated by fewer than $n$ elements. We will show that $M$ is indecomposable.

Let $y_{i}$ and $z_{i}$ be the images of $x_{i}$ in $M$ and $M / \mathfrak{m} M$ respectively. Let $S=a M+b M$. We observe that $a F+b F$ is an $R / m$-vector space with the elements $a x_{1}, \cdots, a x_{n}, b x_{1}, \cdots, b x_{n}$ as a basis. $K$ is a subspace of $a F+b F$ of dimension $n-1$, and $S$ is the corresponding quotient space, with dimension $n+1$. A sample basis for $S$ is $b y_{1}, a y_{1}$, $\cdots, a y_{n}$, from which it follows that $a M$ is a subspace of $S$ of codimension 1 , as is $b M$. There are natural homomorphisms $\alpha, \beta$ taking $M / \mathrm{m} M$ into $S$ given by multiplication by $a$ and $b$ respectively, (so $\left.\alpha\left(z_{i}\right)=a y_{i}\right)$ and these are injective (by dimension count). Now suppose $M=A \oplus B$. Clearly $a M=a A \oplus a B$, and $S=(a A+b A) \oplus(a B$ $+b B)$. Since $a M$ has codimension one in $S$, one of these summands of $S$ is in $a M$, so we may suppose $b B \subseteq a B$. Choose $w=c_{1} z_{1}+\cdots+c_{n} z_{n}$ in $B / \mathrm{m} B$ so as to minimize the index of the first nonzero coefficient. (Note that $B / \mathfrak{m} B \neq 0$ by Nakayama's lemma.) Note that $c_{1}=0$, since otherwise $\beta(w) \notin a M$ (since a basis of $S$ is $b y_{1}, a y_{1}, \cdots, a y_{n}$ and the last $n$ elements generate $a M)$ and we know, in fact, that $\beta(w) \in a B$. If $w=c_{2} z_{2}+\cdots+c_{n} z_{n}$, then $\beta(w)=\alpha\left(c_{2} z_{1}+\cdots+c_{n} z_{n-1}\right)$, so $c_{2} z_{1}$ $+\cdots+c_{n} z_{n-1}$ is in $B / \mathrm{m} B$, contradicting the choice of $w$ (since $\alpha$ is injective). This contradiction shows that $M$ is indecomposable.

CoRollary 2.1. If $R$ is a local ring and every finitely presented module is a summand of a direct sum of cyclic modules, then $R$ is a generalized valuation ring.

Proof. Any indecomposable summand of a direct sum of cyclic modules over a commutative local ring is again a cyclic module by Azumaya's theorem [1], so this follows from Theorem 2.

\section{Global results.}

THEOREM 3. A commutative ring $R$ has the property that every finitely presented module is a summand of a direct sum of cyclic modules if and only if $R_{\mathfrak{m}}$ is a generalized valuation ring for each maximal ideal $\mathfrak{m}$ in $R$.

Proof. If $R$ is such a ring so is $R_{\mathfrak{m}}$, since any finitely presented $R_{\mathfrak{m}}$-module is of the form $M_{\mathfrak{m}}$ for some finitely presented $R$-module $M$. Hence the condition is necessary by the above corollary. Conversely, by [6, Proposition 4] a finitely generated $R$-module $M$ is RDprojective if and only if $M$ is finitely presented and $M_{\mathfrak{m}}$ is RD-projective for each maximal ideal $m$, from which the result follows. 
We note two simple applications of these results. A submodule $A$ of an $R$-module $B$ is called pure (sometimes "strongly pure") if for any $R$-module $F$, the natural map $F \otimes A \rightarrow F \otimes B$ is injective. It is easy to see [6] that this notion of purity coincides with the relative divisibility of $\S 1$ if and only if every finitely presented $R$-module is a summand of a direct sum of CP modules, so we have characterized in Theorem 3 the commutative rings for which these two notions coincide.

Our second remark concerns the existence of indecomposable finitely presented modules. It is not clear that if $R$ is a ring such that for some maximal ideal $\mathrm{m}, R_{\mathfrak{m}}$ is not a generalized valuation ring, then large finitely presented indecomposable modules exist. All one can say immediately is that for any integer $n>0$ there is a finitely presented module $M$ such that in any direct sum decomposition of $M$ at least one of the summands cannot be generated by fewer than $n$ elements.

3. Noetherian rings. We follow Zariski-Samuel [7] in calling a ring a special PIR if it is a commutative local ring with identity whose maximal ideal $m$ is principal and nilpotent. It is easily checked that the following properties of a commutative local Noetherian ring are equivalent:

(a) $\mathfrak{m} / \mathfrak{m}^{2}$ has $R / \mathfrak{m}$-dimension one (where $\mathfrak{m}$ is the maximal ideal);

(b) $R$ is a discrete valuation ring or a special PIR; and

(c) $R$ is a generalized valuation ring.

We will also need the fact that an integral domain $R$ is Dedekind if and only if for each maximal ideal $\mathfrak{m}, R_{\mathfrak{m}}$ is a discrete valuation ring.

TheOREM 4. The following conditions on a commutative Noetherian ring are equivalent.

(i) For every maximal ideal $\mathfrak{m}, \mathfrak{m} / \mathfrak{m}^{2}$ has $R / \mathfrak{m}$-dimension one.

(ii) $R$ is a (finite) product of Dedekind domains and special PIR's.

(iii) For every maximal ideal $\mathfrak{m}, R_{\mathfrak{m}}$ is a discrete valuation ring or a special PIR.

(iv) Every finitely generated $R$-module is a summand of a direct sum of cyclic modules.

Proof. We recall that for any maximal ideal $m$, there is a natural isomorphism between $R / \mathrm{m}^{2}$ and $R_{\mathfrak{m}} / \mathrm{m}^{2} R_{\mathfrak{m}}$, so that (i) and (iii) are equivalent. Theorem 3 implies that (iii) and (iv) are equivalent. Clearly (ii) implies (iii), so it remains to show that (iii) implies (ii).

We assume that $R$ is indecomposable and show that it is a special PIR or an integral domain (necessarily Dedekind). $R$ has only a 
finite number of minimal prime ideals [7, pp. 208-211]. By [7, p. 224, Corollary 1 ] if $m$ is a maximal ideal, there is a one-to-one correspondence between the prime ideals of $R_{\mathfrak{m}}$ and the prime ideals of $R$ contained in $\mathrm{m}$. In particular, if $R_{\mathfrak{m}}$ is a special PIR, $m$ is a minimal prime ideal, while any other maximal ideal contains exactly one minimal prime ideal. It follows that the minimal prime ideals are pairwise comaximal and that any set consisting of powers of distinct minimal prime ideals is pairwise comaximal [7, pp. 176-177]. Let $S$ be the set consisting of

(a) those minimal prime ideals which are not maximal, and

(b) for every maximal ideal $m$ such that $R_{\mathfrak{m}}$ is a special PIR, the ideal $\mathfrak{m}^{k}$, where $k$ is some positive integer such that $\mathfrak{m}^{k} R_{\mathfrak{m}}=0$.

The intersection of the ideals in $S$ is zero since its image in each $R_{\mathfrak{m}}$ is zero. Hence $R$ is the product of the rings $R / q, q \in S$. Since $R$ was assumed indecomposable, it follows that $S$ has only one element, so that either $R$ is a special PIR or the unique minimal prime ideal is zero, so $R$ is an integral domain.

COROLlary 4.1. If $R$ is a commutative Notherian ring then either for every positive integer $n$, there are finitely generated indecomposable $R$-modules which cannot be generated by fewer than $n$ elements, or $R$ satisfies the conditions of Theorem 4, in which case any finitely generated indecomposable $R$-module can be generated by two elements.

Proof. If for some $\mathrm{m}, R_{\mathrm{m}}$ is not a generalized valuation ring, then $R / \mathrm{m}^{2}$ is a local ring which is not a generalized valuation ring, so the result follows from Theorem 2 . Conversely, if $R$ satisfies the conditions of Theorem 4, then any indecomposable $R$-module is essentially a module over one of the indecomposable summands of $R$, and hence is either cyclic or isomorphic to an ideal in one of the Dedekind domains. This proves the result, since any ideal in a Dedekind domain can be generated by two elements.

We close by noting two corollaries of Theorem 4 which were previously known. Theorem 4 is a generalization of the theorem of Krull ([4] or [7, p. 245, Theorem 3]) that a Noetherian ring is a principal ideal ring if and only if it is the product of an Artinian principal ideal ring and a finite number of principal ideal domains. Theorem 4 also contains the result of Uzkov [5] that a commutative Noetherian ring has the property that every finitely generated module is a direct sum of cyclic modules if and only if it is a principal ideal ring. The question of what rings have the property that every finitely presented module is a direct sum of cyclic modules is still unsolved, even for domains. 


\section{BiBLIOGRAPHY}

1. G. Azumaya, Corrections and supplementaries to my paper concerning KrullRemak-Schmidt's theorem, Nagoya Math. J. 1 (1950), 117-124. M R 12, 314.

2. D. Higman, Indecomposable representations at characteristic $p$, Duke Math. J. 21 (1954), 377-381. MR 16, 794.

3. I. Kaplansky, Modules over Dedekind rings and valuation rings, Trans. Amer. Math. Soc. 72 (1952), 327-340. MR 13, 719.

4. W. Krull, Die verschiedenen Arten der Hauptidealringe, S.-B. Heidelberger Akad. Wiss. 6 (1924).

5. A. I. Uzkov, On the decomposition of modules over a commutative ring into direct sums of cyclic submodules, Mat. Sb. 62 (104) (1963), 469-475. (Russian) MR 28 \#1214.

6. R. B. Warfield, Jr., Purity and algebraic compactness for modules, Pacific J. Math. 28 (1969), 699-719.

7. O. Zariski and P. Samuel, Commutative algebra. Vol. 1, The University Series in Higher Math., Van Nostrand, Princeton, N. J., 1958. MR 19, 833.

University of Washington, Seattle, Washington 98105 